\title{
ARTICLE \\ Behavioral and neural markers of cigarette-craving regulation in young-adult smokers during abstinence and after smoking
}

\author{
Dara G. Ghahremani ${ }^{1}$, Paul Faulkner ${ }^{1}$, Chelsea M. Cox ${ }^{1}$ and Edythe D. London ${ }^{1,2,3}$
}

\begin{abstract}
Cigarette craving contributes substantially to the maintenance of tobacco use disorder. Behavioral strategies to regulate craving may facilitate smoking cessation but remain underexplored. We adapted an emotion-regulation strategy, using proximal/distal selfpositioning, to the context of cigarette craving to examine craving regulation in 42 , daily smokers (18-25 years old). After overnight abstinence from smoking, before and after smoking their first cigarette of the day, participants viewed videos of natural scenes presenting young adults who were either smoking cigarettes ("smoke") or not ("non-smoke"). Before each video, participants were instructed to imagine themselves either immersed in the scene ("close") or distanced from it ("far"). They rated their craving after each video. Task-based fMRI data are presented for a subsample of participants $(N=21)$. We found main effects of smoking, instruction, and video type on craving - lower ratings after smoking than before, following the "far" vs. "close" instructions, and when viewing non-smoke vs. smoke videos. Before smoking, "smoke" vs. "non-smoke" videos elicited activation in, orbitofrontal cortex, anterior cingulate, lateral parietal cortex, mid-occipital cortex, ventral striatum, dorsal caudate, and midbrain. Smoking reduced activation in anterior cingulate, left inferior frontal gyrus, and bilateral temporal poles. Activation was reduced in the ventral striatum and medial prefrontal cortex after the "far" vs. the "close" instruction, suggesting less engagement with the stimuli during distancing. The results indicate that proximal/distal regulation strategies impact cue-elicited craving, potentially via downregulation of the ventral striatum and medial prefrontal cortex, and that smoking during abstinence may increase cognitive control capacity during craving regulation.
\end{abstract}

Neuropsychopharmacology (2018) 43:1616-1622; https://doi.org/10.1038/s41386-018-0019-7

\section{INTRODUCTION}

Although the prevalence of cigarette smoking in the U.S. has declined substantially in the past decade [1], the use of combustible tobacco products persists as the leading cause of preventable death and disease in the U.S. [2]. Smoking cessation, therefore, is among the most important health-promoting changes that can reduce the risk of a variety of diseases [3]. Young adults (ages 18-25 years old) represent a substantial proportion of smokers, and focusing cessation efforts on smokers in this age group may result in higher quit rates than in older adults who have had a longer period to establish dependence [4].

One of the primary challenges associated with smoking cessation is in the management of craving and withdrawal symptoms, which help maintain tobacco use. Self-regulation strategies for reducing craving have an important role in potentially disrupting this maintenance and have been examined in the context of cue-induced craving. In such contexts, a variety of both implicit and explicit regulation strategies reduce craving acutely (see ref. [5] [6]) for review. Application of cravingregulation strategies engages frontal cortical and subcortical regions, including the anterior cingulate cortex, medial prefrontal cortex, dorsolateral prefrontal cortex, amygdala, ventral striatum, and the midbrain ([7]; see ref. [8] [9, 10]) for review, and evidence supports the notion that downregulation of craving occurs via prefrontal-striatal interaction [9].
In most studies of craving regulation, participants have been tested during acute abstinence, when craving is heightened. The neural mechanisms involved in regulation of craving likely shift as a consequence of smoking, yet no studies have compared the dynamics of craving regulation between the states of abstinence and after smoking. Comparison of these states may offer insight into the above-mentioned fronto-striatal interactions that modulate the craving response. To make this comparison, we obtained behavioral and functional MRI (fMRI) measures of craving regulation in young adult smokers after overnight abstinence $(\geq 12 \mathrm{~h})$, before and after they smoked their first cigarette of the day (of their preferred brand). We expected fronto-striatal activation related to craving regulation to diminish from before to after smoking, assuming that reduced craving after smoking a cigarette will result in less engagement of neural mechanisms to regulate craving.

Craving-regulation strategies used in most studies require explicit, effortful downregulation of craving. Such strategies are somewhat open-ended with minimal constraint over the participant's strategic method, potentially leading to significant variability of behavioral and neural data within and between participants. To address this variability, we adapted a strategy that has been used in the emotion-regulation literature [11-13] and invokes a proximal/distal framing in which participants are instructed to imagine themselves either immersed in a scene

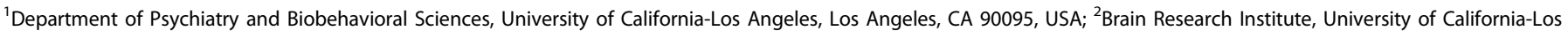
Angeles, Los Angeles, CA 90095, USA and ${ }^{3}$ Department of Molecular and Medical Pharmacology, University of California-Los Angeles, Los Angeles, CA 90095, USA Correspondence: Dara G. Ghahremani (darag@ucla.edu)

Received: 17 August 2017 Revised: 18 January 2018 Accepted: 20 January 2018

Published online: 5 February 2018 
("close") or at a distance from it ("far"). Instead of static images of smoking-related cues, to evoke a tangible, natural, and dynamic context of smoking, we used well-controlled video clips of young adults in scenes showing them either smoking or not. Self-report ratings of craving were collected after each video presentation. We expected an interaction of close/far instruction and video type (smoke/non-smoke), with the greatest craving occurring during the close, smoke condition. A sample of 42 participants completed the behavioral paradigm, and a subsample $(N=27)$ performed the same task during fMRI.

\section{MATERIALS AND METHODS}

Participants

Participants were young adults (18-25 years of age), who reported smoking $\geq 5$ cigarettes per day for $\geq 1$ year. They were recruited using internet and print media advertisements. Exclusion criteria were: positive urine test for illicit drugs (including marijuana) on test days, endorsing smoking marijuana $>8$ times per month or consuming alcohol on $>15$ days per month, use of psychotropic medications, any DSM-IV Axis I psychiatric disorder other than nicotine dependence, as assessed by the Mini International Neuropsychiatric Interview [14], desire for treatment for tobacco use disorder, history of neurological injury, current pregnancy, lefthandedness (so as to not introduce variation in brain imaging), preference for menthol cigarettes, or use of nicotine products other than cigarettes (e.g., electronic cigarettes, chewing tobacco).

Overall, 42 young adults participated in the study, and 27 of them underwent fMRI scanning. Three of the fMRI participants did not complete the study, leading to insufficient data, and three participants were excluded from $\mathrm{fMRI}$ analyses due to excessive head motion during scanning ( $>2 \mathrm{~mm}$ translational displacement, $>1.5^{\circ}$ rotation). In total, behavioral data from 42 participants, and fMRI data from 21 of these participants were included in analyses. Participants received compensation in the form of cash.

\section{Procedures}

Screening and characterization of participants. All procedures were approved by the UCLA Office for the Protection of Research Subjects. Participants gave written informed consent after receiving a detailed explanation of the study. Then they underwent eligibility screening using questionnaires, psychiatric interview questions, and urine toxicology. Self-reports regarding prior drug use were obtained using a standardized questionnaire. Participants were required to demonstrate recent smoking by providing breath samples that had CO concentrations $>10 \mathrm{ppm}$, measured using a portable monitor (coVita, Haddonfield, NJ) and urine samples with cotinine concentrations $>3 \mathrm{ng} / \mathrm{ml}$ (NicAlert, Nymox Corporation, Hasbrouck Heights, NJ). Women provided urine samples to test for pregnancy, which was exclusionary. Clinical features related to tobacco use disorder were obtained using the Fagerström Test for nicotine dependence (FTND) [15].

\section{Scan day procedures}

Participants who satisfied the eligibility requirements of the study were required to remain abstinent from smoking for at least $12 \mathrm{~h}$, verified by $\mathrm{CO}$ levels $(<10 \mathrm{ppm})$ in expired air on days of testing. Urine tests negative for illicit substances were also required. Participants were scanned before and after smoking their first cigarette of the day, a cigarette of their preferred brand, $\sim 35 \mathrm{~min}$ before performing the task in the scanner. Scanning sessions were conducted at the same time of day (abstinence and post-smoking scans at $\sim 10 \mathrm{AM}$ and $12 \mathrm{PM}$, respectively). Participants were scanned on five separate days as part of a larger study that examined the effects of smoking four research cigarettes delivering different doses of nicotine in addition to conventional (preferred brand) cigarettes, across five different testing sessions (results partially reported elsewhere, e.g., [16]). Testing days were spaced by an average of

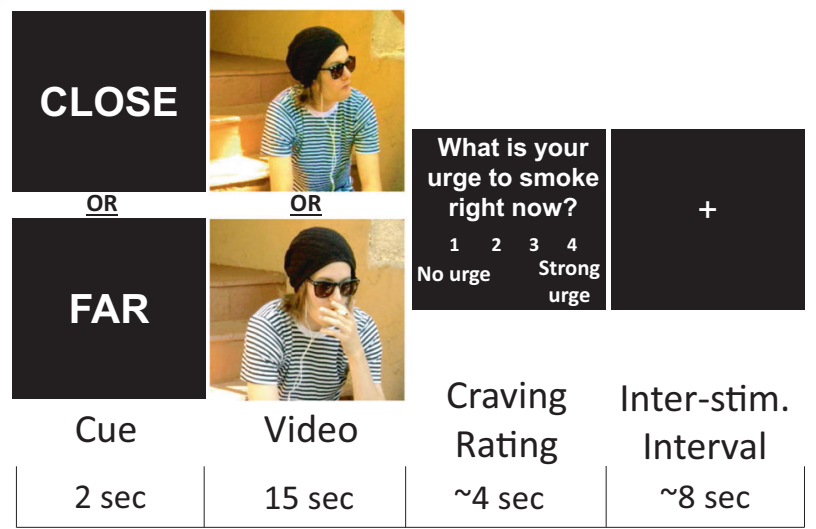

Fig. 1 Schematic of proximal/distal craving-regulation fMRI task. Participants were presented with an instructional cue ("close" or "far") indicating whether they should imagine themselves having proximal or distal disposition (see Materials and methods) to the scene depicted in the subsequently presented video clip. After presentation of the video, participants rated their craving on a scale of 1-4. Participants had up to $4 \mathrm{~s}$ to respond. After making a button press, their choice was highlighted on the screen ( $1 \mathrm{~s}$ ) followed by the inter-stimulus interval (ISI) that preceded the subsequent trial. A fixation cross appeared on the screen during the ISI for an average period of $8 \mathrm{~s}$ (see Materials and methods for further details)

5.22 days $(S D=4.32)$, and participants returned to smoking-as-usual between test days. Data reported here only include those from the abstinence scan on each of the 5 days and after smoking on one day (randomized across participants), when they smoked their preferred brand cigarette. Non-scanned participants underwent all of the same procedures as the scanned group except that the craving-regulation task was performed in a testing room outside of the scanner and presented on a laptop computer.

\section{Craving-regulation task}

The task performed during scanning (Fig. 1) was modeled after an incidental regulation task that uses proximal/distal perspective taking as an approach to regulate affect $[12,13]$ or food craving [11]. In the task used here, participants viewed videos of young adults in natural settings (e.g., waiting at a bus stop) while smoking a cigarette ("smoke" condition) or not ("non-smoke" condition). Prior to each video presentation (15s), participants were given one of two instructions, "close" or "far" (2 s). On "close" trials, participants were instructed to imagine themselves immersed in the scene depicted in the video and to allow themselves to experience any sensations (e.g., imagined smells) potentially evoked by the video. On "far" trials, participants were told to imagine themselves as neutral observers, standing at a distance from the scene, and to make factual, objective observations of the content of the scene (e.g., indoors/outdoors). Following video presentation, participants were asked to rate their urge to smoke ("How much do you feel like smoking?") on a fourpoint Likert scale, with a rating of "one" equivalent to "not at all" and "four" representing "very much", with their right hand using a four-button button box in the scanner for scanned participants and the numbers 1-4 on a laptop keyboard for non-scanned participants. Participants had up to $4 \mathrm{~s}$ to respond on each trial. After making a button press, their choice was highlighted on the screen $(1 \mathrm{~s})$, followed by presentation of a fixation cross for $8 \mathrm{~s}$. Five such trials were administered in a practice session outside the scanner before the session began. Twenty-four trials in two blocks (scanning runs) were administered. Although participants were not told so, "close" trials were used to assess baseline cuereactivity, whereas the difference between craving ratings in the "close" and "far" trials were used to assess regulation. 
The video clips used in the task were created by a professional film crew using several professional young-adult actors depicted in various scenes (e.g., at a kitchen table; waiting at a bus stop), either by themselves or in interaction with each other. Importantly, "smoke" and "non-smoke" videos were matched in all aspects of content, except for whether the actors were smoking or not. The videos did not include audio. Thirty-six unique 30-s videos were created. Each of these videos was split into two 15-s clips to accommodate the number of trials needed for the study. Three sets of 24 videos were used in the study. Each video clip was only presented once across abstinent and post-smoking sessions. Counterbalancing procedures were used to ensure that participants viewed equivalent video content across pre-smoking scans and post-smoking scans (e.g., same actors, scenes).

The presentation and timing of all stimuli and response events were programmed using Matlab (Mathworks, Natick, MA) and the Psychtoolbox (www.psychtoolbox.org) on an Apple MacBook Pro laptop running Mac OSX (Apple Computers, Cupertino, CA). During scanning, visual stimuli were presented using a projector at the rear of the bore of the scanner, with participants viewing them via a mirror mounted on the head coil.

\section{Brain imaging}

Imaging was performed using a 3-T Siemens AG (Erlangen, Germany) Trio MRI scanner with a 32-channel coil. We acquired two runs of 222 functional T2*-weighted echoplanar images (EPI) (slice thickness, $4 \mathrm{~mm}$; 34 slices; repetition time (TR), $2 \mathrm{~s}$; echo time (TE), $30 \mathrm{~ms}$; flip angle, $90^{\circ}$; matrix, $64 \times 64$; field of view (FOV), 192 $\mathrm{mm}$ ). Three additional volumes were discarded at the beginning of each run to allow for $\mathrm{T} 1$ equilibrium effects. For registration purposes, a T2-weighted matched bandwidth high-resolution anatomical scan (same slice prescription as EPI) and a magnetization-prepared rapid-acquisition gradient echo (MPRAGE) high-resolution scan (slice thickness, $1 \mathrm{~mm}$; 176 slices per slab; TR, $2530 \mathrm{~s}$; TE, $3.31 \mathrm{~ms}$; flip angle, $7^{\circ}$; matrix, $256 \times 256$; FOV, $256 \mathrm{~mm}$; sagittal orientation) were acquired for each participant. The orientation for matched bandwidth and EPI scans was oblique axial to maximize full brain coverage and to optimize signal from ventral prefrontal regions.

\section{Data analysis}

Behavioral data. Mean craving ratings from each participant were submitted to a generalized linear mixed model with participant as random effect using the Ime4 software package [17] within the R statistical programming language and environment (www.r-project.org). With craving rating as the dependent variable, task instruction ("close"/"far"), video type ("smoke"/"nonsmoke"), and smoking ("pre/post") were independent variables of interest. Sex was included as a covariate. Age was not included as a covariate due to the narrow age range of the participants $(M=$ 22.3, $S D=2.2$ ). Separate models were run for the entire sample and the subset of participants who had fMRI scans.

Imaging data. Analysis of $\mathrm{fMRI}$ data was performed using the FSL (5.0.9) toolbox from the Oxford Centre for fMRI of the Brain (www. fmrib.ox.ac.uk/fsl). Procedures for analyses are described in detail in the supplementary materials. Briefly, image preprocessing included registration to compensate for head motion, skullremoval, spatial smoothing, and spatial registration to standard space (Montreal Neurological Institute (MNI) avg152 template). Whole-brain, voxel-wise statistical analyses were performed using a multi-stage approach to implement a mixed-effects model, treating participants as a random effects variable. Each of the four task conditions were modeled as separate regressors. Motion parameters were included as covariates of no interest to account for variance associated with residual motion.

To examine trial-by-trial relationships between self-reported craving and fMRI activation, we performed a separate analysis in
Table 1. Results from generalized linear mixed-effects model testing associations between task conditions and craving ratings during task performance

\begin{tabular}{|c|c|c|c|c|}
\hline & Estimate & SE & $t$ & $p$ \\
\hline \multicolumn{5}{|l|}{ Craving ratings $(N=42)$} \\
\hline \multicolumn{5}{|l|}{ Abstinence only ${ }^{\mathrm{a}}$} \\
\hline Instruction ("close"/"far") & 1.73 & 0.32 & 5.36 & $<0.001$ \\
\hline Cue ("smoke"/"nonsmoke") & -0.22 & 0.06 & -3.95 & $<0.001$ \\
\hline Instruction $\times$ cue & -0.10 & 0.06 & -1.78 & 0.08 \\
\hline Sex & 0.30 & 0.20 & 1.46 & 0.14 \\
\hline Day & -0.02 & 0.01 & -1.62 & 0.16 \\
\hline \multicolumn{5}{|l|}{ Effect of smoking ${ }^{a}$} \\
\hline Instruction ("close"/"far") & 2.27 & 0.37 & 6.14 & $<0.001$ \\
\hline Cue ("smoke"/"nonsmoke") & -0.26 & 0.06 & -4.29 & $<0.001$ \\
\hline Smoking & 0.85 & 0.06 & 14.01 & $<0.001$ \\
\hline Sex & -0.32 & 0.06 & -5.36 & 0.1 \\
\hline Day & 0.31 & 0.19 & 1.66 & 0.12 \\
\hline \multicolumn{5}{|l|}{ Craving ratings $(N=21)$} \\
\hline \multicolumn{5}{|l|}{ fMRI only ${ }^{a}$} \\
\hline Instruction ("close"/"far") & -0.37 & 0.08 & -4.49 & $<0.001$ \\
\hline Cue ("smoke"/"nonsmoke") & 0.78 & 0.13 & 6.07 & $<0.001$ \\
\hline Smoking & -0.18 & 0.08 & -2.23 & 0.03 \\
\hline Sex & 0.31 & 0.22 & 1.42 & 0.16 \\
\hline Day & -0.03 & 0.10 & -0.28 & 0.78 \\
\hline
\end{tabular}

which a parametric modulation covariate [18] was added, indicating each participant's response for each trial during abstinence. To compute the overlap of cue-induced craving (smoke vs. non-smoke cues) and these parametric modulation results, we conducted a statistical conjunction analysis using methods described in Nichols et al. [19] with a height threshold of $Z=2.3$.

For all analyses, time-series statistical analysis was carried out using linear modeling with local autocorrelation correction [20] after highpass temporal filtering. Contrast images for runs within each scanning session were combined using a fixed effects analyses. Abstinence scans were combined across the 5 days of scanning in separate fixed effects models for each subject in which day was included as a covariate of no interest to account for potential practice or habituation effects. To determine effects of smoking (pre- to post), pairwise, fixed effects analyses comparing contrast images from the two sessions were first conducted for each subject. These images were then submitted for group analyses using random effects analyses.

For group analyses, the FMRIB Local Analysis of Mixed Effects (FLAME1) module in FSL was used [21, 22]. Z (Gaussianised T) statistic images were thresholded using cluster-corrected statistics with a height threshold of $Z>2.3$ and a cluster probability threshold of $p<0.05$, whole-brain corrected using the theory of Gaussian random fields [23]. All group analyses were subjected to robust outlier deweighting [24]. Sex was included as a covariate of no interest. Anatomical locations of activations were identified using the Harvard-Oxford Probabilistic Atlas and the sectional brain atlas of Duvernoy and Bourgouin [25].

\section{RESULTS}

Characteristics of research participants: demographics and cigarette use

The 42 participants included in the behavioral analyses were 18-25 years of age $(M=22.3, S D=2.2 ; 21$ female). The ethnic/ racial composition was $47.6 \%$ Caucasian, $9.5 \%$ Hispanic/Latino, 
16.6\% African-American, and 14.3\% Asian, with two participants indicating two categories (Asian/Hispanic and African-American/ Hispanic). Participants reported smoking 5-40 cigarettes per day $(M=11.6, \mathrm{SD}=6.2)$ and had a mean FTND score of $3.54(\mathrm{SD}=2.03)$. All participants had at least a high school education.

The subset of 21 participants who completed fMRI scanning and were included in analyses were 19-25 years of age $(M=22.6, \mathrm{SD}=$ 2.0; 10 female). The ethnic/racial composition was $57 \%$ Caucasian, 24\% Hispanic/Latino, 19\% African-American, and 10\% Asian, including the two participants that indicated two categories (Asian/Hispanic and African-American/Hispanic). Participants reported smoking 5-20 cigarettes per day $(M=11.1, \mathrm{SD}=4.8)$ and had a mean FTND score of $3.2(\mathrm{SD}=1.75)$.

Task performance. Behavioral results for the full sample $(N=42)$ from the full-factorial model indicated no significant interactions between the independent variables. Removing the interaction

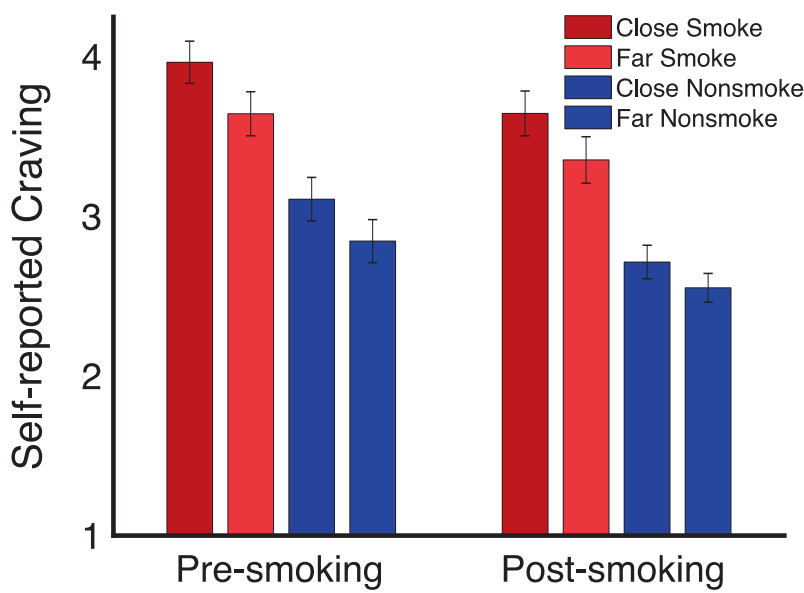

Fig. 2 Mean craving ratings given after each video presentation for each task condition before and after smoking a cigarette for the entire sample $(N=42)$. Main effects of smoking cue, proximal/distal instruction, and cigarette smoking were observed; no significant interactions were found. Errors bars reflect one standard error of the mean terms from the model revealed main effects of instruction (close/ far), video type (smoke/nonsmoke videos), and smoking on cue-elicited craving-lower craving ratings were given after vs. before smoking, following the "far" vs. "close" instructions, and when viewing the nonsmoke vs. the smoke videos (Table 1; Fig. 2). The same pattern of results was observed in the subsample of participants who received fMRI $(N=21)$ (Figure S1). No significant differences were observed with respect to environment (fMRI or out of scanner; no main effects or interactions, all Ps $>0.1$ ). No significant main or interaction effects of sex were observed in either the fMRI sample or the larger behavioral sample.

\section{fMRI results}

Brain activation related to cue-induced reactivity before smoking: When participants viewed videos containing smoking-related vs. neutral stimuli, activation was observed in the medial orbitofrontal cortex, posterolateral orbital frontal cortex, ventral striatum extending into the ventral anterior insula, dorsal anterior caudate, bilateral lateral mid-occipital cortex, bilateral posterior lateral parietal cortex, and midbrain (Fig. 3, hot colors; Table S1). Greater activation for non-smoking vs. smoking cues was observed along the medial wall of the occipital cortex (calcarine and lingual cortices) and bilateral somatosensory cortex (Fig. 3, cool colors; Table S1). Although sex was included in the model as a covariate of no interest and the sample sizes for each group were relatively small (10 female, 11 male), we note that females showed greater activation for the smoking vs. non-smoking videos than males in caudate, thalamus, anterior cingulate cortex, and left superior/middle frontal gyrus (Figure S6). We also note that differences in activation for smoking vs. non-smoking videos were located within brain regions that showed positive activation to videos in general (not within regions that showed "deactivations" to videos).

Task-related brain activation modulated by trial-by-trial craving ratings before smoking: Results of the parametric modulation analysis indicated that activation in rostral anterior cingulate, medial prefrontal cortex, lateral orbitofrontal cortex, posterior cingulate, dorsal and ventral striatum, and lateral occipital cortex during video presentations (both types) was modulated by participants' craving ratings (Figure S2; Table S1). Many of these

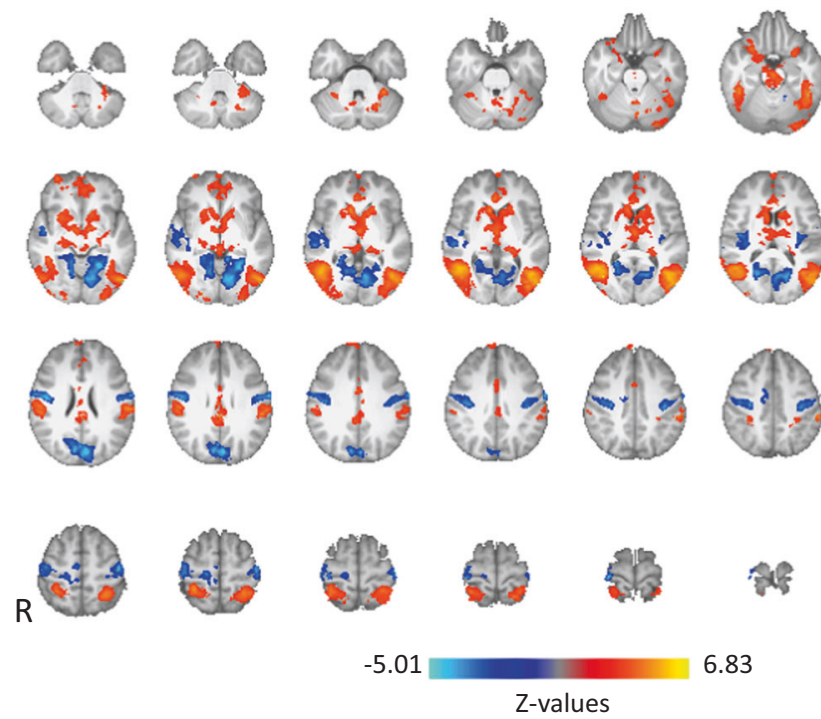

Fig. 3 Main effect of smoking cues (smoking vs. non-smoking cues) during abstinence. Activation related to smoking vs. non-smoking cues are presented in hot colors. The reverse contrast, non-smoking vs. smoking cues, are presented in cool colors. Image shows thresholded Zstatistic map overlaid on a group-averaged high-resolution anatomical image. $\mathrm{R}=$ right (images are displayed in radiological orientation; right is left). Color bar indicates Z-statistic range. 
regions overlapped with those found for the contrast of smoking vs. non-smoking cues (see Figure S3 for conjunction analysis; Table S1).

Brain activation related to craving regulation before smoking. When participants viewed videos (both those that contained smoking cues and those that did not) under the "close" vs. "far" instruction (i.e., main effect of instruction), greater activation was observed in the ventral striatum and bilateral anterior superior frontal cortex (Fig. 4; Table S1). No supra-threshold activation was observed for the reverse contrast ("far" vs. "close"). We also did not observe relationships between this contrast and behavioral indices of regulation (far-close craving ratings).

Effects of smoking on activation related to cue-induced reactivity. Comparison of cue-induced activation (smoking vs. non-smoking videos) before vs. after smoking revealed activation in rostral and caudal anterior cingulate cortex, including the subgenual segment, left inferior frontal gyrus, and bilateral superior and middle temporal gyri (Fig. 5; Table S1). To examine the direction of this interaction of pre/post smoking by cue (smoking vs. non-smoking videos), we extracted model parameter estimates from the four clusters of activation. As shown in Figure S4, the interaction was primarily driven by a smoking-related reduction in activation for smoking cues and not non-smoking cues. No regions showed supra-threshold activation when comparing activation after vs. before smoking (i.e., no regions showed significantly increased activation due to smoking a cigarette).

Effects of smoking on activation related to craving regulation. Examination of effects of smoking on activation related to craving regulation did not reveal any supra-threshold voxels in wholebrain analysis.

Effects of smoking severity. We did not find any relationships between cigarettes per day (CPD) $(M=11.67, \quad S D=6.15)$, a measure of smoking severity, and craving ratings during the task (no main effects of cue-type or instruction-type, or interactions). In a whole-brain group-level analysis, in which sex and CPD were included as covariates, we found no effect of CPD on the cueinduced craving contrast (smoke vs. non-smoke cues) and craving regulation (close vs. far).
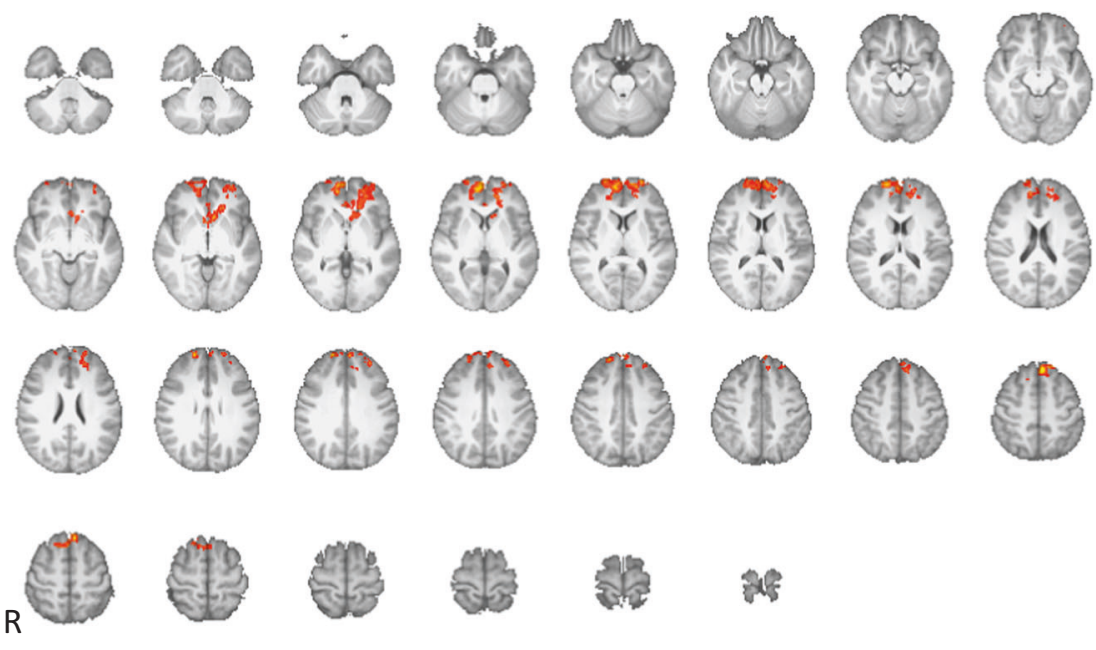

2.3

$$
\text { Z-values }
$$

4.12

Fig. 4 Main effect of proximal/distal instruction (close vs. far) during abstinence. No supra-threshold clusters were observed for the reverse contrast, far vs. close. Image shows thresholded Z-statistic map overlaid on a group-averaged high-resolution anatomical image. $\mathrm{R}=$ right (images are displayed in radiological orientation; right is left) Color bar indicates Z-statistic range

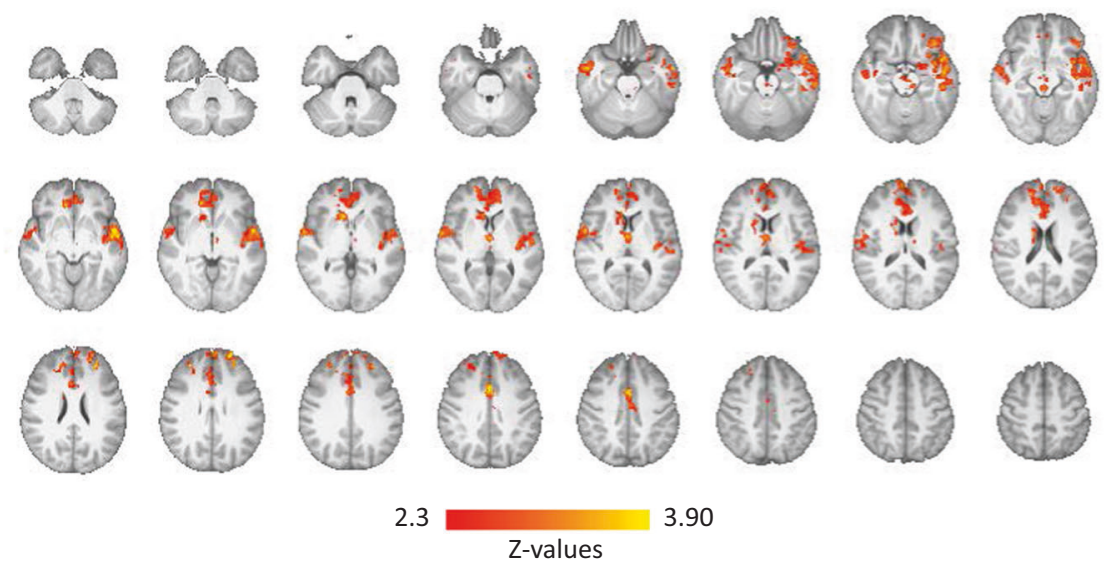

Fig. 5 Effect of smoking on smoking cue-induced activation. Comparison of cue-induced activation (smoking- vs. non-smoking cues) during abstinence vs. after smoking a cigarette. Image shows thresholded Z-statistic map overlaid on a group-averaged high-resolution anatomical image. $\mathrm{R}=$ right (images are displayed in radiological orientation; right is left). Color bar indicates $Z$-statistic range. 


\section{DISCUSSION}

Use of a craving-regulation fMRI task employing a proximal/distal manipulation revealed main effects of smoking cues, regulation, and smoking on craving in young-adult smokers. That is, less craving was reported after viewing non-smoke vs. smoke cues, after the far instruction than the close condition, and after smoking than before. Consistent with previous meta-analyses of neuroimaging studies of cue-induced craving [26, 27], the medial prefrontal cortex, ACC, lateral occipital/ventral temporal cortex, and ventral striatum were responsive to smoking-related cues (relative to non-smoking cues) during abstinence. A subset of these regions (i.e., medial prefrontal cortex, ACC, and ventral striatum) exhibited decreased activation after smoking. Regions that had activation correlated with trial-by-trial ratings of craving showed strong overlap with those showing activation for smoke vs. non-smoke cues, indicating that these cue-related activations were indeed related to the state of craving. Craving-regulation effects were observed in medial prefrontal cortex (mostly, superior frontal gyrus (SFG)) and ventral striatum, and no changes in activation related to craving regulation were observed as a result of smoking.

Results from this study indicate that mentally distancing oneself from smoking-related environmental stimuli can reduce cigarette craving and are in line with results from studies, which used the same distancing strategy in the context of emotion regulation, such that imagined distance confers reduced negative feelings [11-13]. Moreover, we found that this distancing strategy not only reduced craving when smoking cues were present, but also during presentation of neutral (non-smoke) cues (i.e., videos in which the same scenes were presented but without the actors smoking). Although these scenes did not contain smoking-related stimuli, it is possible that the contextual cues from the same scenes in which smoking stimuli were present produced some carry-over effects, resulting in further reduction in craving when the distancing strategy was applied.

Neuroimaging studies that have examined self-regulation in response to appetitive cues have observed decreases in activation in ventral striatum and orbitofrontal cortex and increases in lateral prefrontal cortex and anterior cingulate cortex when participants are asked to explicitly down-regulate their response ([7]; see ref. [28] [9]) for review. Our observation of reduced ventral striatum activation with distancing from smoking cues is consistent with these findings; however, with respect to the involvement of the prefrontal cortex, we only observed activation changes (decreases) in SFG, not in ventral prefrontal areas (including the OFC) and lateral PFC. The lack of observed activation changes in these areas in our study may be due to the differences in task demands between distancing and explicit self-regulation strategies. Effortful re-appraisal strategies for explicit downregulation of an appetitive response may involve several cognitive processes, including reappraisal strategies in which appetitive qualities of the cue are transiently devalued (e.g., imagining that the object is fake or laced with poison). Such strategies require cognitive control, typically involving lateral PFC regions [29], and often recruit OFC, a region involved in representation of value [30]. By simply adjusting one's imagined spatial disposition to the stimulus, distancing does not involve re-appraisal of the stimulus, which may include its devaluation. Although inference regarding behavioral processes from brain activation (or lack there of) must be considered with caution [31], it is likely that we did not observe activation in such prefrontal regions due to the characteristics of the task that differ from explicit self-regulation tasks.

Our observation of decreased SFG activation with distancing supports prior results indicating a role for this region in modulating craving. A previous study using repetitive transcranial magnetic stimulation showed that high frequency stimulation of SFG induced increased craving in response to smoking cues [32]. We show that a manipulation of one's spatial disposition to smoking cues not only reduces craving, but also reduces activation in a region shown to causally modulate craving. SFG has extensive connections to the striatum [33], suggesting that the ventral striatal reductions we observed during regulation was in coordination with SFG. Moreover, we observed reduction in cueinduced activation in the area of SFG after smoking a cigarette, providing further evidence for the role of this region in modulation of craving. Although most explorations of brain stimulation for treatment of nicotine dependence have targeted lateral PFC (e.g., [34]), these results suggest that further work is needed to determine the role of more medial areas, such as SFG, that may be specifically important for regulation of craving.

Our results indicate that smoking a cigarette does not influence craving regulation. Smoking a cigarette reduced craving over all, but no change in the magnitude of craving regulation was observed, as indicated by the lack of interaction between smoking and task instruction. Combined with the fact that smoking did not result in changes in brain activation related to craving regulation, these results suggest that smoking itself does not necessarily change the capacity for regulation (at least in the form of distancing under examination here) or affect the neural systems associated with it. However, given that distancing is likely less effortful than explicit cognitive reappraisal strategies, one possibility is that more effortful forms of self-regulation would be influenced by smoking. More studies are required to test this hypothesis.

Our study focused on young adult smokers (ages 18-25) for several reasons. Smoking cessation before the age of 25 can deter most of the negative health consequences of smoking [35]. Therefore, reducing the prevalence of smoking among youth can have a significant impact on improving public health [36]. Young smokers display different smoking behaviors compared to older smokers-they smoke fewer cigarettes per day and exhibit lower nicotine dependence than older adult smokers, and only transition from light, intermittent smoking to heavy, daily smoking between ages 20 and 25 [37]. Further, smokers in this age range are still undergoing brain development: myelination and synaptic pruning in the brain continue into the third decade of life, determining the ultimate gray-matter density of the frontal lobe and its connections to subcortical structures [38, 39]. As such, the neural mechanisms of craving, withdrawal and their relief from smoking may differ for young, more inexperienced smokers compared to older, experienced smokers. Moreover, given the reliance on selfregulation strategies on function of the prefrontal cortex, young smokers may engage this still-developing area of the brain differently than older smokers. While our study did not include a comparison group that differed in developmental stage (e.g., adults older than 25) to test unique attributes of this developmental population, our results indicate that smokers in this age group are able to utilize regulation strategies, such as distancing, to successfully modulate craving, likely via SFG-ventral striatal coordination.

The current study is not without limitations. The voxel-height threshold chosen for the cluster-based statistics employed in making inferences about of the fMRI data $(Z>2.3)$ has been shown to be less than optimal across several fMRI analysis packages [40]. However, we note that in Eklund and colleagues' analyses, FSL's FLAME1, used here, performed better than other software packages with the threshold of $Z>2.3$ (or $p<0.01$ ) for analyses that used event-related designs, falling within the range of a $95 \%$ confidence interval for most analyses. Nevertheless, nonparametric statistics or higher statistical thresholds would have been preferred assuming a larger sample size than used here. Practice, habituation or, more generally, test-retest effects, are difficult to rule out with the experimental design employed; however, we took several precautions to minimize these effects. First, we used different videos across abstinence and postsmoking scans, counterbalancing them across participants. Second, to evaluate potential test-retest effects on craving independent of smoking, we included an additional day of 
behavioral sessions for five participants in which they completed all aspects of the protocol except for smoking a cigarette. This small sample showed a trend towards increased craving from time 1 to time 2 (Figure S5), suggesting that the smoking-related decreases in craving we observed were highly likely related to the effects of smoking and less likely due to habituation to the stimuli or practice in performing the task.

Overall, this study suggests that distancing may be a viable, implicit behavioral strategy for reducing craving with this reduction occurring via fronto-striatal involvement-SFG and ventral striatum, in particular. Moreover, smoking a cigarette, thereby reducing craving, does not have an impact on craving regulation as measured by distancing, nor does it impact the neural mechanisms associated with it. Although further work is needed, this study may have important clinical implications for inclusion of craving-regulation strategies in behavioral treatments and for providing targets for neurotherapeutic interventions, such as brain stimulation. Such strategies may be particularly relevant and effective for treatment of nicotine addiction among young adults.

\section{ACKNOWLEDGEMENTS}

Funding was provided by the US National Institute on Drug Abuse (R01 DA036487-02 to EDL). Additional funding was provided by an endowment from the Thomas P. and Katherine K. Pike Chair in Addiction Studies (to EDL) and a gift from the Marjorie M. Greene Trust. Preliminary results from the current study were presented at the Society for Neuroscience Annual Meeting in 2015.

\section{ADDITIONAL INFORMATION}

Supplementary information accompanies this paper at https://doi.org/10.1038/ s41386-018-0019-7.

Conflict of interest: The authors declare that they have no conflict of interest.

\section{REFERENCES}

1. Centers for Disease Control and Prevention. Current Cigarette Smoking Among Adults in the U.S. 2016. https://www.cdc.gov/tobacco/data_statistics/fact_sheets/ adult_data/cig_smoking/index.htm. Accessed August 3rd, 2017

2. Britton J. Death, disease, and tobacco. Lancet. 2017;389:1861-2.

3. Sasco AJ, Secretan MB, Straif K. Tobacco smoking and cancer: a brief review of recent epidemiological evidence. Lung Cancer. 2004;45:S3-9.

4. Messer K, Trinidad DR, Al-Delaimy WK, Pierce JP. Smoking cessation rates in the United States: a comparison of young adult and older smokers. Am J Public Health. 2008;98:317-22.

5. Jasinska AJ, Stein EA, Kaiser J, Naumer MJ, Yalachkov Y. Factors modulating neural reactivity to drug cues in addiction: a survey of human neuroimaging studies. Neurosci Biobehav Rev. 2014;38:1-16.

6. Kober $\mathrm{H}$, Kross EF, Mischel W, Hart CL, Ochsner KN. Regulation of craving by cognitive strategies in cigarette smokers. Drug Alcohol Depend. 2010;106:52-55.

7. Brody AL, Mandelkern MA, Olmstead RE, Jou J, Tiongson E, Allen V, et al. Neural substrates of resisting craving during cigarette cue exposure. Biol Psychiatry. 2007;62:642-51.

8. Kober $\mathrm{H}$, Mell MM. Neural mechanisms underlying craving and the regulation of craving. In: Wilson SJ, editor. The Wiley handbook on the cognitive neuroscience of addiction. Oxford, UK: John Wiley \& Sons, Ltd; 2015. pp 195-218.

9. Kober H, Mende-Siedlecki P, Kross EF, Weber J, Mischel W, Hart CL, et al. Prefrontal-striatal pathway underlies cognitive regulation of craving. Proc Natl Acad Sci USA. 2010;107:14811-6.

10. Zhao L-Y, Tian J, Wang W, Qin W, Shi J, Li Q, et al. The role of dorsal anterior cingulate cortex in the regulation of craving by reappraisal in smokers. PLoS ONE. 2012;7:e43598.

11. Silvers JA, Insel C, Powers A, Franz P, Weber J, Mischel W, et al. Curbing craving: behavioral and brain evidence that children regulate craving when instructed to do so but have higher baseline craving than adults. Psychol Sci. 2014;25:1932-42.

12. Silvers JA, McRae K, Gabrieli JDE, Gross JJ, Remy KA, Ochsner KN. Age-related differences in emotional reactivity, regulation, and rejection sensitivity in adolescence. Emotion. 2012;12:1235-47.

13. Silvers JA, Shu J, Hubbard AD, Weber J, Ochsner KN. Concurrent and lasting effects of emotion regulation on amygdala response in adolescence and young adulthood. Dev Sci. 2015;18:771-84.
14. Sheehan DV, Lecrubier $Y$, Sheehan KH, Amorim P, Janavs J, Weiller E, et al. The Mini-International Neuropsychiatric Interview (M.I.N.I.): the development and validation of a structured diagnostic psychiatric interview for DSM-IV and ICD-10. J Clin Psychiatry. 1998;59:22-33. quiz 34-57

15. Fagerstrom K, Russ C, Yu CR, Yunis C, Foulds J. The Fagerstrom test for nicotine dependence as a predictor of smoking abstinence: a pooled analysis of varenicline clinical trial data. Nicotine Tob Res. 2012;14:1467-73.

16. Faulkner P, Ghahremani DG, Tyndale RF, Cox CM, Kazanjian AS, Paterson N, et al. (2017). Reduced-nicotine cigarettes in young smokers: impact of nicotine metabolism on nicotine dose effects. Neuropsychopharmacology. 2017;42:1610-18.

17. Bates $D$, Maechler M, Bolker B, Walker S. Linear mixed-effects models using Eigen and S4. R Package Version. 2014;1:Ime4.

18. Buchel C, Holmes AP, Rees G, Friston KJ. Characterizing stimulus-response functions using nonlinear regressors in parametric fMRI experiments. Neurolmage. 1998;8:140-8.

19. Nichols T, Brett $M$, Andersson J, Wager T, Poline J-B. Valid conjunction inference with the minimum statistic. Neurolmage. 2005;25:653-60.

20. Woolrich MW, Ripley BD, Brady M, Smith SM. Temporal autocorrelation in univariate linear modeling of FMRI data. Neuroimage. 2001;14:1370-86.

21. Beckmann CF, Jenkinson M, Smith SM. General multilevel linear modeling for group analysis in FMRI. Neuroimage. 2003;20:1052-63.

22. Woolrich MW, Behrens TE, Beckmann CF, Jenkinson M, Smith SM. Multilevel linear modelling for FMRI group analysis using Bayesian inference. Neuroimage. 2004;21:1732-47.

23. Worsley KJ, Evans AC, Marrett S, Neelin P. A three-dimensional statistical analysis for CBF activation studies in human brain. J Cereb Blood Flow Metab. 1992;12:900-18.

24. Woolrich M. Robust group analysis using outlier inference. Neuroimage. 2008;41:286-301.

25. Duvernoy HM, Bourgouin P. The Human brain: surface, three-dimensional sectional anatomy with MRl, and blood supply. Wien; New York: Springer; 1999. p. 491.

26. Engelmann JM, Versace F, Robinson JD, Minnix JA, Lam CY, Cui Y, et al. Neural substrates of smoking cue reactivity: a meta-analysis of fMRI studies. NeuroImage. 2012;60:252-62.

27. Chase HW, Eickhoff SB, Laird AR, Hogarth L. The neural basis of drug stimulus processing and craving: an activation likelihood estimation meta-analysis. Biol Psychiatry. 2011;70:785-93.

28. Kelley WM, Wagner DD, Heatherton TF. In search of a human self-regulation system. Annu Rev Neurosci. 2015;38:389-411.

29. Buhle JT, Silvers JA, Wager TD, Lopez R, Onyemekwu C, Kober H, et al. Cognitive reappraisal of emotion: a meta-analysis of human neuroimaging studies. Cereb Cortex. 2014;24:2981-90.

30. Rushworth MFS, Kolling N, Sallet J, Mars RB. Valuation and decision-making in frontal cortex: one or many serial or parallel systems? Curr Opin Neurobiol. 2012;22:946-55.

31. Poldrack RA. Can cognitive processes be inferred from neuroimaging data? Trends Cogn Sci. 2006;10:59-63.

32. Rose JE, McClernon FJ, Froeliger B, Behm FM, Preud'homme X, Krystal AD. Repetitive transcranial magnetic stimulation of the superior frontal gyrus modulates craving for cigarettes. Biol Psychiatry. 2011;70:794-9.

33. Croxson PL, Johansen-Berg H, Behrens TE, Robson MD, Pinsk MA, Gross CG, et al. Quantitative investigation of connections of the prefrontal cortex in the human and macaque using probabilistic diffusion tractography. J Neurosci. 2005;25:8854-66.

34. Dinur-Klein L, Dannon P, Hadar A, Rosenberg O, Roth Y, Kotler M, et al. Smoking cessation induced by deep repetitive transcranial magnetic stimulation of the prefrontal and insular cortices: a prospective, randomized controlled trial. Biol Psychiatry. 2014;76:742-9.

35. Doll R, Peto R, Boreham J, Sutherland I. Mortality in relation to smoking: 50 years' observations on male British doctors. BMJ. 2004;328:1519.

36. Benowitz NL, Henningfield JE. Establishing a nicotine threshold for addiction. The implications for tobacco regulation. N Engl J Med. 1994;331:123-5.

37. White HR, Bray BC, Fleming CB, Catalano RF. Transitions into and out of light and intermittent smoking during emerging adulthood. Nicotine Tob Res. 2009;11:211-9.

38. Giedd JN, Raznahan A, Alexander-Bloch A, Schmitt E, Gogtay N, Rapoport JL. Child psychiatry branch of the National Institute of Mental Health longitudinal structural magnetic resonance imaging study of human brain development. Neuropsychopharmacology. 2015;40:43-49.

39. Sowell ER, Thompson PM, Tessner KD, Toga AW. Mapping continued brain growth and gray matter density reduction in dorsal frontal cortex: Inverse relationships during postadolescent brain maturation. J Neurosci. 2001;21:8819-29.

40. Eklund A, Nichols TE, Knutsson $H$. Cluster failure: Why fMRI inferences for spatial extent have inflated false-positive rates. Proc Natl Acad Sci USA. 2016;113:7900-5. 